\title{
Fully automated quantification of cardiac chamber and function assessment in 2-D Echocardiography: clinical feasibility of deep learning-based algorithms
}

\section{Sekeun Kim ( $\sim$ sk.k@yonsei.ac.kr)}

Yonsei University https://orcid.org/0000-0003-4196-6242

\section{Hyung-Bok Park}

Catholic Kwandong University International Saint Mary's Hospital

Jaeik Jeon

Connect-Al Research Center

Reza Arsanjani

Mayo Clinic Arizona

\section{Ran Heo}

Hanyang University Seoul Hospital

\section{Sang-Eun Lee}

Ewha Womans University Seoul Hospital

Inki Moon

Soonchunhyang University Hospital Bucheon

\section{Sun Kook Yoo}

Yonsei University College of Medicine

Hyuk-Jae Chang

Yonsei University College of Medicine

\section{Research Article}

Keywords: echocardiography, deep learning, fully automated

Posted Date: September 30th, 2021

DOl: https://doi.org/10.21203/rs.3.rs-945163/v1

License: (c) (1) This work is licensed under a Creative Commons Attribution 4.0 International License. Read Full License

Version of Record: A version of this preprint was published at The International Journal of Cardiovascular Imaging on February 13th, 2022. See the published version at https://doi.org/10.1007/s10554-021- 
02482-y.

Page 2/21 


\section{Abstract}

Objectives: We aimed to compare the segmentation performance of the current prominent deep learning $(\mathrm{DL})$ algorithms with ground-truth segmentations and to validate the reproducibility of the manually created 2D echocardiographic four cardiac chamber ground-truth annotation.

Background: Recently emerged DL based fully-automated chamber segmentation and function assessment methods have shown great potential for future application in aiding image acquisition, quantification, and suggestion for diagnosis. However, the performance of current DL algorithms have not previously been compared with each other. In addition, the reproducibility of ground-truth annotations which are the basis of these algorithms have not yet been fully validated.

Methods: We retrospectively enrolled 500 consecutive patients who underwent transthoracic echocardiogram (TTE) from December 2019 to December 2020. Simple U-net, Res-U-net, and Dense-U-net algorithms were compared for the segmentation performances and clinical indices such as left atrial volume (LAV), left ventricular end diastolic volume (LVEDV), LV end systolic volume (LVESV), LV mass, and ejection fraction (EF) were evaluated. The inter- and intra- observer variability analysis was performed by two expert sonographers for a randomly selected echocardiographic view in 100 patients (apical 2-chamber, apical 4-chamber, and parasternal short axis views).

Results: The overall performance of all DL methods was excellent (average Dice similarity coefficient (DSC) 0.91 to 0.95 and average Intersection over union (IOU) 0.83 to 0.90 ), with the exception of LV wall area on PSAX view (average DSC of 0.83 , IOU 0.72). In addition, there were no significant difference in clinical indices between ground truth and automated DL measurements. For inter- and intra observer variability analysis, the overall intra observer reproducibility was excellent: LAV (ICC $=0.995)$, LVEDV (ICC $=0.996)$, LVESV $(I C C=0.997)$, LV mass $(I C C=0.991)$ and EF $(I C C=0.984)$. The inter-observer reproducibility was slightly lower as compared to intraobserver agreement: LAV (ICC $=0.976)$, LVEDV (ICC $=0.982)$, LVESV $(I C C=0.970)$, LV mass $(I C C=0.971)$, and EF $(I C C=0.899)$.

Conclusions: The three current prominent DL-based fully automated methods are able to reliably perform four-chamber segmentation and quantification of clinical indices. Furthermore, we were able to validate the four cardiac chamber ground-truth annotation and demonstrate an overall excellent reproducibility, but still with some degree of inter-observer variability.

\section{Introduction}

Echocardiography is a primary cardiac imaging tool which provides noninvasive real-time imaging for identifying cardiac structure along with function in clinical practice. However, unlike other imaging modalities such as computed tomography (CT) or magnetic resonance imaging (MRI), echocardiography is highly operator dependent with various types of artifacts which can occur during image acquisition as well as post-processing [1]. Unavoidably, these limitations can cause large inter and intraobserver variability and poor reproducibility, resulting in clinical shortcomings, in particular, when it comes to 
monitoring longitudinal quantitative measurements [2,3]. Even though, semi-automated delineation and quantification of cardiac structures have demonstrated their utility in the quantitative echocardiographic assessment, they still need significant amount of manual modification which is time-consuming and may introduce the risk of the inter-/intra-observer variability [4].

The adoption of artificial intelligence (Al) technology in echocardiographic imaging has recently emerged as a novel alternative solution for these challenges $[5,6]$. With the advance of deep neural networks, there have been attempts to develop fully-automated algorithms for mainly focusing on the left ventricle (LV), where there is a clinical unmet need for the accuracy and reproducibility of quantitative assessments [7]. Importantly, in order to develop an accurate chamber segmentation deep learning algorithm, which is the basis of the functional assessment, is dependent on the ground-truth image data, being so called 'training data set', being essentially accurate and reproducible $[8,9]$.

The poor repeatability is primarily due to the low signal-to-noise ratio, edge dropout, and presence of shadow, there is a high variability in quantitative assessment of echocardiography [2, 3]. However, to date, there has been no investigation of the inter-/intra-observer variability of ground-truth of four chamber data itself which is crucial for the accurate and advanced deep-learning algorithms. In addition, there have been no comparisons among currently available deep learning algorithms to suggest the most potent approaches for fully automated quantitative analysis nor have there been clinical performance comparisons made between the algorithms and conventional techniques.

Therefore, firstly we sought to evaluate whether currently prominent deep learning methods could accurately estimate clinical indices including LV mass, LV/LA volume, and ejection fraction when compared mutually as well as with conventional methods. Secondly, we validated the reproducibility of our manually created ground-truth segmentations in two-dimensional echocardiography which are imperative for the development of highly advanced deep-learning Al based algorithms.

\section{Methods}

\section{Study population}

In this study, we retrospectively enrolled consecutive patients who underwent transthoracic echocardiogram (TTE) from December 2019 to December 2020. The Institutional Review Board at Yonsei University Severance Cardiovascular Hospital approved this study protocol. Inclusion criteria consisted of having had all echocardiography visually correspond to standard views and we excluded patients with diagnosis of heart failure, coronary artery disease, valvular heart disease, known pregnant state, uninterpretable quality of echocardiographic images or images that were acquired by non-standardized scan angles, and those had abnormal echocardiograms (Table.1). All patients were scanned in the left lateral position using grayscale second-harmonic 2D imaging techniques, with the adjustment of image contrast, frequency, depth, and sector size for adequate frame rate and optimal LV border visualization. All patients received a complete quantification report, which was validated by cardiologists. Echocardiographic images were acquired by standard ultrasound equipment including Vivid 9 (GE 
Healthcare, Horton, Norway; $n=259)$, EPIQ 7C (Phillips Healthcare, Andover, MA, USA; $n=170)$, Acuson SC2000 (Siemens, Mountain View, CA, USA; $n$ = 42), and Artida (Canon Medical Systems, Tokyo, Japan; $n$ $=29$ ).

\section{Ground-truth generation}

Anonymized echocardiographic digital images were analyzed and annotated in a core laboratory, where experienced sonographers (five expert sonographers who all had more than 5 years of echocardiographic experience) manually contoured cardiac structures according to the recommendations of the American Society of Echocardiography [10]. For each patient, we selected a set of B-mode images including apical two chamber (A2C) view, apical four chamber (A4C) view and parasternal short axis (PSAX) view at papillary muscle (PM) level which included at least one cardiac cycle. In a series of multi-video frames, manual annotation at end-diastole (ED) and end-systole (ES) for four chambers were performed using a commercial annotation tool (OsiriX, Pixmeo, Switzerland). Two chambers including left ventricle (LV) and left atrium (LA) were delineated when available in A2C, A4C, and PSAX views, respectively. In addition, we further delineated four chamber structures including right ventricle (RV) and right atrium (RA) in $A 4 C$ view (Figure 1). During LV endocardial wall segmentation, trabeculations and papillary muscles were excluded in PSAX view at PM level.

\section{Inter- and intra-observer reproducibility analysis}

To validate our manually created ground truth annotation, we performed both inter- and intra- observer variability analysis. Two sonographers, who both had more than 5 years of echocardiography experience with more than 5,000 echocardiographic examinations, were chosen and the variability of clinical indices were assessed on a randomly selected echocardiographic images of A2C, A4C, and PSAX views in 100 patients.

One sonographer manually annotated LV, LA, RV, and RA contours on the three views at end diastole (ED) and end systole (ES) with an interval of one month with a randomly shuffled analysis preventing the observer from being influenced by previous measurements (intra-observer variability). The other sonographer annotated the same groups without being influenced by the other (inter-observer variability).

\section{Dataset splitting}

A total of 500 echocardiograms were allocated for developing automated deep learning methods. Each of the echocardiograms consisted of consecutive frames with dozens of still images. All information was removed from echocardiograms that could identify individual patients. Echocardiographic images were extracted from anonymized DICOM files, and unorganized videos with different views were grouped according to their views. The entire dataset containing 500 patients was divided into training $(80 \%, n=$ $400)$, validation $(10 \%, n=50)$, and test set $(10 \%, n=50)$ for deep learning methods. The 5 -fold cross validation was employed to analyze the generalization performance of ML methods. The entire dataset containing 500 patients was divided into five groups. In training stages, four subsets were used for 
training and validation of the network. In test stages, the remainder was employed to evaluate the ML model.

\section{Deep Learning-Based Algorithms}

To automatically segment cardiac structures in echocardiography, we employed three deep learning models based on U-net which were used for biomedical image segmentation and have demonstrated high performance on segmentation of organs [11]. The U-net consists of a fully convolutional encoder path, called backbone with a symmetric expanding decoder path for segmentation [11]. We constructed three deep learning models on the basis of encoder-decoder architecture of U-net. In our experiments, we deployed the backbone of U-net architecture with residual and dense blocks, which shows robust performance in the image classification network with a U-net decoder path $[12,13]$. The flow chart of deep learning methods is shown in Figure 2.

\section{Training strategy}

Given input images and corresponding annotated masks containing different categories with their views, data augmentation was performed with up-down, left-right flips, and rotation. For training deep learning models, a pixel-wise cross-entropy loss function was employed to minimize inaccuracy of prediction. An Adam optimizer with learning rate of 0.0001 was adopted to optimize network parameter. We trained our models from scratch without using any pretrained weight for initialization. We randomly shuffled training dataset and trained deep network for 200 epochs with a mini-batch size of 5. All input cases were resized to $512 \times 512$ due to GPU memory limitation and then the image intensity values were normalized into the range of $[0,1]$.

\section{Performance analysis of deep-learning models}

Dice similarity coefficient (DSC) and Intersection over union (IOU), and clinical indices such as volume, mass, and ejection fraction (EF) were included to compare the performance of deep learning methods. The DSC and IOU are both quantifies the pixel-wise degree of similarity between the model-predicted segmentation mask and the ground truth, and ranges from 0 (no similarity) to 1 (identical). We employed segmentation results of the deep learning model to calculate clinical index to compare chamber structure quantification and ejection fraction based on standard guidelines. Left and right volume were calculated by the area-length method derived by the long axis and area of A2C and A4C [10]. LVM was calculated as the left ventricular myocardial volume derived by the delineation of its endocardial and epicardial borders and multiplied with the specific gravity of myocardial tissue (assuming a tissue density of $1.05 \mathrm{~g} / \mathrm{ml}$ ). These annotations, which were established and verified by board-certified cardiologists, were used as the 'ground truth' for the deep-learning model.

\section{Statistical analysis}

Continuous and normally distributed variables were represented as mean \pm SD and median with interquartile range (IQR) for non-normally distributed variables. Comparison between ground truth and 
prediction results were assessed by the paired t test and Pearson correlation coefficient using two-sided $p$ values. A p value of $<0.05$ was considered significant. Bland-Altman plots with $95 \%$ confidence intervals for correlation were calculated. Inter-observer and intra-observer reproducibility were assessed by the intraclass correlation coefficients (ICC) for absolute agreement of single measures between two observers. All statistical analyses were performed using commercially available statistics software (MedCalc, version 18.9, MedCalc software Inc., Mariakerke, Belgium).

\section{Results}

\section{Baseline patient characteristics}

Baseline characteristics of subjects are described in Table 1. The mean age was $36.2 \pm 12.6$ years, and $50.2 \%$ of the population were male. The mean Body mass index was $22.2 \pm 2.9$, and Body surface area was $1.7 \pm 0.19$. The average of LVEF was $67.0 \pm 4.9$ and LV mass was $132.8 \pm 32.7$, and LA volume was $43.3 \pm 10.7$, respectively. Among patients analyzed, 215 (43\%) had hypertension, $110(22 \%)$ had diabetes, and 235 (47\%) had dyslipidemia. The patients' echocardiographic were obtained using various vendors including GE Healthcare (51.8\%), Philips Healthcare (34.0\%), Siemens (8.4\%), and Canon Medical Systems (5.8\%).

\section{Performance comparison between deep-learning models}

In table 2, automated segmentation performances between deep neural networks (U-net vs. Res-U-net vs. Dense-U-net) were compared by using DSC and IOU metrics. Except LV wall area on PSAX PM level view (average DSC of 0.83, IOU 0.72 ), all deep learning methods showed an overall excellent performance (average DSC 0.91 to 0.95 and average IOU 0.83 to 0.90 ) (Table 2 and Figure 3). There were no significant differences observed between the three deep learning methods for all the parameters analyzed at each echocardiographic view (Table 2).

We also compared commonly used clinical indices such as LA volume (LAV), LV end diastolic volume (LVEDV), LV end systolic volume (LVESV), LV mass, and EF. There were no significant differences found between ground truth and automated ML measurements (Table 3). The correlation and Bland-Altman plots for these indices are presented in Figure 4. Overall, the model prediction for all structures correlated well with no significant differences as compared with manual ground truth annotation: LAV $(r=0.90 ; p<$ $0.001)$, LVEDV $(r=0.88 ; p<0.001)$, LVESV $(r=0.81 ; p<0.001)$, LV mass $(r=0.79 ; p<0.001)$, and EF $(r=$ $0.67 ; p<0.001)$. Bland-Altman analysis demonstrated a mean difference, especially in EF, which is the ratio of LVEDV and LVESV, showing that accumulation of variability resulted in high variability. However, the difference between manual and automated analysis were not statistically significant.

On the other hand, according to the quality of echocardiographic images, automated deep learning algorithm showed lower performance generally in those with 'poor' image quality as compared to 'good' or 'fair', especially in LV myocardium (Poor 0.70 vs. Fair 0.83 vs. Good 0.84 ) and LV cavity (Poor 0.87 vs. Fair 0.94 vs. Good 0.94) (Table 4 and Figure 5). 
Additionally, we tested the impact of the number of training dataset for robustness of ML algorithm. There were linear association between the performance of automated ML analysis and the increment of dataset until $60 \%$ of the training dataset ( $n=300 ; 1,271$ frames) achieving already the maximal DSC and IOU of 0.93 and 0.87 . After this point, the accuracy was sustained regardless of the number of training dataset (Figure 6).

\section{Inter- and intra-observer variability of ground truth annotation}

Intra observer variability highly correlated for all of the measurements as follows: left atrium volume: ICC $=0.995, p<0.001$; left ventricle diastolic volume; ICC $=0.996, p<0.001$, left ventricle systolic volume; ICC $=$ $0.997, p<0.001$, left ventricular mass; ICC $=0.991, p<0.001$; and ejection fraction; ICC $=0.984, p<0.001$ (Table 5). Inter observer variability showed slightly lower correlation compared to interobserver variability, as follows: left atrium volume: ICC $=0.976, p<0.001$; left ventricle diastolic volume; $I C C=0.982, p<0.001$, left ventricle systolic volume; ICC $=0.970, p<0.001$, left ventricular mass; ICC $=0.971, p<0.001$; and ejection fraction; $I C C=0.889, p<0.001)$. The variability of ejection fraction, which is the difference ratio between LVEDV and LVESV, showed the lowest correlation among clinical metrics (Table 5).

\section{Discussion}

In this study, we were able to show that the current prominent deep learning-based methods can be reliably utilized for automated quantification of cardiac chambers in 2-D echocardiography with no significant segmentation performance variabilities between these algorithms. Simple U-net, Res-U-net, and Dense-U-net are currently the most prominent deep learning algorithms for image segmentation and have demonstrated their robust performance on organ segmentation in biomedical imaging [11-13]. However, we observed that the segmentation performance was substantially influenced by image quality especially involving certain parameters. In addition, we also validated the reproducibility of our manually created four chamber ground-truth annotations which are the fundamental basis of advanced deep learning algorithms, which had not been specifically investigated in prior studies. To our knowledge, this is the first study comparing current prominent deep learning algorithms and validating reproducibility of the four-chamber ground-truth. Furthermore, this is the largest manually annotated datasets acquired in different vendors which was then utilized for the evaluation of automated analysis of echocardiography.

A major well known limitation of quantitative analysis in clinical practice is the significant inter- and intraobserver variability involving tracing of endocardial borders [2, 3]. Even though semiautomated software was used, inherent low reproducibility impedes the reliable longitudinal quantitative assessment, particularly in the following situations: poor image quality scans, patients who have arrhythmias, complex abnormality in cardiac chambers such as congenital anomaly, multivalvular heart disease, presence of regional wall motion abnormalities in the left ventricle due to myocardial infarction, and infiltrative myocardial diseases $[14,15]$. In addition, the relatively time-consuming quantification process prohibits quantification of multiple frames or dynamic frame-by-frame analysis in order to average clinical measurements especially for patients with irregular heartbeats [16]. 
However, recently introduced fully automated deep learning-based cardiac chamber quantification techniques have no operator variability, providing almost real-time quantitative processing $(0.12 \mathrm{sec}$ per frame with CPU), as well as enabling dynamic cardiac chamber quantification $[14,16]$. Zhang et al. nicely demonstrated the feasibility and high diagnostic accuracy of U-Net deep learning-based segmentation algorithms and represented automated view classification with disease detection models, which could be a profound pipeline work for the realization of Al-based one button whole echocardiography quantitative analysis in the future [14]. However, in their study, segmentation and quantitative analysis performances were not tested in poor or modest image quality in various disease models which are frequently encountered in clinical practice. In addition, a relatively small number of ground-truth annotations were used at each view for training their deep learning algorithm from 124 to 214, which might not be sufficient for the development of robust segmentation algorithm. In addition, no validation of reproducibility for ground-truth was presented [14]. In our study, on the other hand, 500 ground-truth annotations were used in deep learning training and analyzed algorithm performances according to image quality. We found that in those with image quality, relatively low segmentation performance were observed for LV cavity, LA cavity, LV myocardium area, which is consistent with previously reported studies $[14,17,18]$.

For the reliability of training data sets, we reported the interobserver and intraobserver variability analysis from 100 patients ( $20 \%$ of total population), demonstrating that inter-observer variability was higher than intra-observer variability showing still some degree of variability exists between experienced experts. Another interesting finding was that high variance was shown in ejection fraction due to the accumulated error from EDLV and ESLV which has constantly shown in prior studies $[14,18]$. Narang et al. introduced a $\mathrm{ML}$ based quantification of 3D echocardiography and compared it to cardiac magnetic resonance imaging [16]. Although small number of patients $(n=20)$ were studied, their accurate and instant frameby-frame LV and LA volume-time curve generation based on 3D segmentation has potential application for searching novel dynamic parameters predicting future outcome [16].

The most significant barrier of deep learning method for echocardiography is the lack of large datasets. Recently, Arafati et al. [17] suggested additional adversarial training to fully convolutional networks to overcome data dependency of network and combined post processing procedure to improve the segmentation performance, but they also reported the variance of segmentation performance depending on age and gender in LV and LA segmentation, respectively. Thus, there are still several unsolved issues that need to be investigated for the full implementation of deep learning-based fully automated quantification into clinical practice. First, further advancement of deep learning based-segmentation algorithms should be made not only for relatively normal heart model but also for various disease models. These algorithms will also need to prove their robustness in studies with modest to poor image qualities (Figure 7). Furthermore, similar to other imaging modalities, inherent operator variation of ground-truth itself is inevitable and the variability will naturally increase depending on image quality and the complexity of heart disease. Therefore, it is essential to diminish this variability and enhance the accuracy of segmentation algorithm through building up large number of accurately annotated groundtruth by experienced echocardiographic experts, despite is being time-consuming and labor-intensive. 
In our study, we represented the relationship between the number of training datasets and segmentation performance showing that maximal performance level was already reached when $60 \%$ of the dataset was being utilized. Still, in order to establish that amount of datasets, it took 5 experienced experts working full time for three months. Moreover, to our knowledge, there has been no study up to now that has validated deep learning methods on the patients with different types of disease. Deep learning models can be improved by diverse sampled data without the class imbalance of disease types, which requires large databases to improve their robust accuracy $[17,19]$.

The recently emerged deep active learning $(A L)$ method could be the solution for this unmet need for the clinical annotation process [20-22]. AL is a powerful method that enables data-efficient model. It reduces the laborious and expensive annotation tasks by carefully choosing data points for which ground-truth need to be labeled [23]. Specifically, AL selects the set of the most informative data points from a pool of unlabeled dataset, so it enables us to minimize the amount of data points that should be labeled and achieve comparable performance to a model trained with fully annotated dataset [24,25].

Echocardiography has been established as an important primary screening test since the incidence of cardiovascular disease has been on the rise [26]. The non-invasive bedside approach enables clinicians to easily monitor the disease progression as well as the response to treatment [26-28]. Furthermore, by using the serial assessment of quantitative parameters including global or longitudinal LV strain, it is possible to diagnose the disease at its preclinical stage and inhibit its progression [29-31]. The development of technology has allowed for the minimalization of echocardiographic systems such as portable handheld transthoracic echocardiography which can further broaden the utility of echocardiography in emergency settings by novice or non-expert paramedics [32-34]. However, the operator dependency of echocardiography particularly in achieving quantitative parameters serves as a significant limitation. Recently emerged deep learning-based artificial intelligence technologies may allow us to level the playing field between rural and urban areas through Al aid or guided fully automated image acquisition and quantification. These novel methods can also be widely adopted in busy echocardiographic laboratories as well as be deployed in emergency departments for more efficient ways to triage patients.

\section{Study Limitations}

Our study has several limitations. These data should be interpreted cautiously because of its retrospective single-center study design; hence, further prospective multi-center studies are warranted. In addition, various types of disease models were not included in our analysis. However, for the first time, we evaluated the performance of fully automated deep learning-based methods according to image quality as well as the required number of training datasets to achieve the maximal performance. Therefore, our study results provided important considerations for future studies for developing specific disease model algorithms. Finally, we only included four chambers on limited echocardiographic views in our analysis. However, to establish a fully automated quantification, beyond the four cardiac chambers, aorta, valves, and pericardial structures should be automatically included into quantification. 
Also, instant multiple frame-by-frame segmentation method is needed to obtain average quantitative parameters in patients with irregular heartbeats.

\section{Conclusion}

We demonstrated that three current prominent deep learning-based fully automated methods are all reliable to perform four-chamber segmentation and quantification of clinical indices without any superiority. However, the performances of these methods were significantly dependent on image quality as well as the number of well annotated training datasets. Additionally, this is the first study to validate the reproducibility of the four cardiac chamber ground-truth annotation itself showing overall excellent reproducibility, but some degree of inter-observer variability still being noted. This study emphasizes that further technical advancement of the fully automated deep learning methods is needed to maintain the clinical performance even in low quality image and specific disease models, not only that, well-designed clinical validation studies are desperately warranted for these technologies to be applied into clinical practice.

\section{Declarations}

No potential conflict of interest was reported by the authors.

\section{Tables}


Table 1. Baseline clinical characteristics. $(n=500)$

\begin{tabular}{ll}
\hline & $\mathrm{n}$ \\
\hline Age (years) & $36.2 \pm 12.6$ \\
\hline Male, $\mathrm{n}(\%)$ & $251(50.2)$ \\
\hline Height $(\mathrm{cm})$ & $167.1 \pm 8.6$ \\
\hline Weight $(\mathrm{kg})$ & $62.2 \pm 11.3$ \\
\hline Body mass index $(\mathrm{kg} / \mathrm{m} 2)$ & $22.2 \pm 2.9$ \\
\hline Body surface area & $1.7 \pm 0.19$ \\
\hline Systolic BP $(\mathrm{mmHg})$ & $119.4 \pm 15.3$ \\
\hline Diastolic BP (mmHg) & $74.2 \pm 11.4$ \\
\hline Hypertension, $\mathrm{n}(\%)$ & $215(43)$ \\
\hline Diabetes, $\mathrm{n}(\%)$ & $110(22)$ \\
\hline Dyslipidemia, $\mathrm{n}(\%)$ & $235(47)$ \\
\hline Vendors, $\mathrm{n}(\%)$ & \\
\hline GE Healthcare & $259(51.8)$ \\
\hline Philips Healthcare & $170(34.0)$ \\
\hline Siemens & $42(8.4)$ \\
\hline Canon Medical Systems & $29(5.8)$ \\
\hline LVEF & $67.0 \pm 4.9$ \\
\hline LV mass & $132.8 \pm 32.7$ \\
\hline LA volume & $43.3 \pm 10.7$ \\
\hline LA volume index & $25.4 \pm 5.4$ \\
\hline
\end{tabular}

Table 2. Performance of each deep neural network (rows) in A4C and PSAX. Error measures described as mean and std computed between ground truth and results. 


\begin{tabular}{|c|c|c|c|c|c|c|}
\hline View & Segmented Area & Metric & U-net & Res-U-net & Dense-U-net & Avg. \\
\hline \multirow[t]{8}{*}{$\mathrm{A} 4 \mathrm{C}$} & \multirow[t]{2}{*}{ LV cavity } & DSC & 0.96 & 0.95 & 0.92 & 0.94 \\
\hline & & IOU & 0.92 & 0.91 & 0.86 & 0.90 \\
\hline & \multirow[t]{2}{*}{ LA cavity } & DSC & 0.94 & 0.92 & 0.95 & 0.94 \\
\hline & & IOU & 0.88 & 0.86 & 0.91 & 0.88 \\
\hline & \multirow[t]{2}{*}{ RV cavity } & DSC & 0.91 & 0.91 & 0.90 & 0.91 \\
\hline & & IOU & 0.87 & 0.86 & 0.83 & 0.85 \\
\hline & \multirow[t]{2}{*}{ RA cavity } & DSC & 0.93 & 0.92 & 0.92 & 0.92 \\
\hline & & IOU & 0.84 & 0.83 & 0.83 & 0.83 \\
\hline \multirow[t]{4}{*}{$\mathrm{A} 2 \mathrm{C}$} & \multirow[t]{2}{*}{ LV cavity } & DSC & 0.95 & 0.95 & 0.95 & 0.95 \\
\hline & & IOU & 0.90 & 0.90 & 0.90 & 0.90 \\
\hline & \multirow[t]{2}{*}{ LA cavity } & DSC & 0.93 & 0.92 & 0.92 & 0.92 \\
\hline & & IOU & 0.87 & 0.85 & 0.85 & 0.86 \\
\hline \multirow[t]{4}{*}{ PSAX } & \multirow[t]{2}{*}{ LV cavity } & DSC & 0.95 & 0.95 & 0.95 & 0.95 \\
\hline & & 100 & 0.90 & 0.91 & 0.91 & 0.90 \\
\hline & \multirow[t]{2}{*}{ LV myocardium } & DSC & 0.83 & 0.82 & 0.84 & 0.83 \\
\hline & & IOU & 0.72 & 0.71 & 0.73 & 0.72 \\
\hline
\end{tabular}

Table 3. Comparison of clinical indices between automated and ground truth from two-dimensional echocardiography. 


\begin{tabular}{|c|c|c|c|}
\hline & Median value (IQ & & $p$ \\
\hline & Ground truth & Automated ML & \\
\hline & & (Simple U-Net) & \\
\hline Left atrium volume, $\mathrm{ml}$ & 48.01 & 48.90 & 0.3172 \\
\hline & $(40.02-54.48)$ & $(42.91-52.55)$ & \\
\hline Left ventricle diastolic volume, $\mathrm{ml}$ & 121.11 & 117.21 & 0.2628 \\
\hline & $(105.22-128.42)$ & $(104.45-125.41)$ & \\
\hline Left ventricle systolic volume, $\mathrm{ml}$ & 55.48 & 58.75 & 0.1584 \\
\hline & $(48.86-63.31)$ & $(51.33-65.12)$ & \\
\hline Left ventricle mass, $\mathrm{g}$ & 199.98 & 203.43 & 0.1741 \\
\hline & $(169.84-242.86)$ & $(172.24-224.34)$ & \\
\hline Ejection fraction & 61.60 & 56.82 & 0.0797 \\
\hline & $(51.50-71.56)$ & $(52.46-63.43)$ & \\
\hline
\end{tabular}

Table 4. Comparison of performance with image quality in DSC.

\begin{tabular}{|llllll|}
\hline View & Segmented Area & Poor & Fair & Good & avg \\
\hline A2C & LV cavity & 0.87 & 0.94 & 0.94 & 0.94 \\
\cline { 2 - 6 } & LA cavity & 0.82 & 0.92 & 0.89 & 0.95 \\
\hline \multirow{2}{*}{ A4C } & LV cavity & 0.95 & 0.93 & 0.96 & 0.97 \\
\cline { 2 - 6 } & LA cavity & 0.93 & 0.90 & 0.92 & 0.91 \\
\cline { 2 - 6 } & RV cavity & 0.85 & 0.87 & 0.90 & 0.92 \\
\cline { 2 - 6 } & RA cavity & 0.91 & 0.89 & 0.91 & 0.92 \\
\hline PSAX & LV cavity & 0.91 & 0.95 & 0.95 & 0.93 \\
\cline { 2 - 6 } & LV myocardium & 0.70 & 0.83 & 0.84 & 0.83 \\
\hline
\end{tabular}

Table 5. Reproducibility of the analysis of each structure. 


\begin{tabular}{|lllll|}
\hline Clinical index & $\begin{array}{l}\text { Intra observer } \\
\text { variability ICC } \\
(95 \% \mathrm{Cl})\end{array}$ & $p$ & $\begin{array}{l}\text { Inter observer variability ICC } \\
(95 \% \mathrm{Cl})\end{array}$ & $p$ \\
\hline Left atrial volume & $\begin{array}{l}0.995(0.993- \\
0.997)\end{array}$ & $<0.001$ & $0.976(0.964-0.984)$ & $<0.001$ \\
\hline $\begin{array}{l}0.996(0.993- \\
\text { Left ventricle end diastolic }\end{array}$ & $<0.001$ & $0.982(0.973-0.988)$ & $<0.001$ \\
\hline $\begin{array}{l}0.997) \\
\text { Lelume }\end{array}$ & & & $<0.001$ \\
\hline volume & $\begin{array}{l}0.997(0.995- \\
\text { Left ventricular mass }\end{array}$ & $<0.001$ & $0.970(0.955-0.980)$ & $<0.001$ \\
\hline $\begin{array}{l}0.998) \\
\text { Left ventricular ejection }\end{array}$ & $\begin{array}{l}0.991(0.984) \\
\text { faction }\end{array}$ & $<0.984(0.976-$ & $0.971(0.956-0.980)$ & $<0.001$ \\
\hline
\end{tabular}

ICC intraclass correlation, $\mathrm{Cl}$ confidence interval

\section{Figures}




\section{Image}
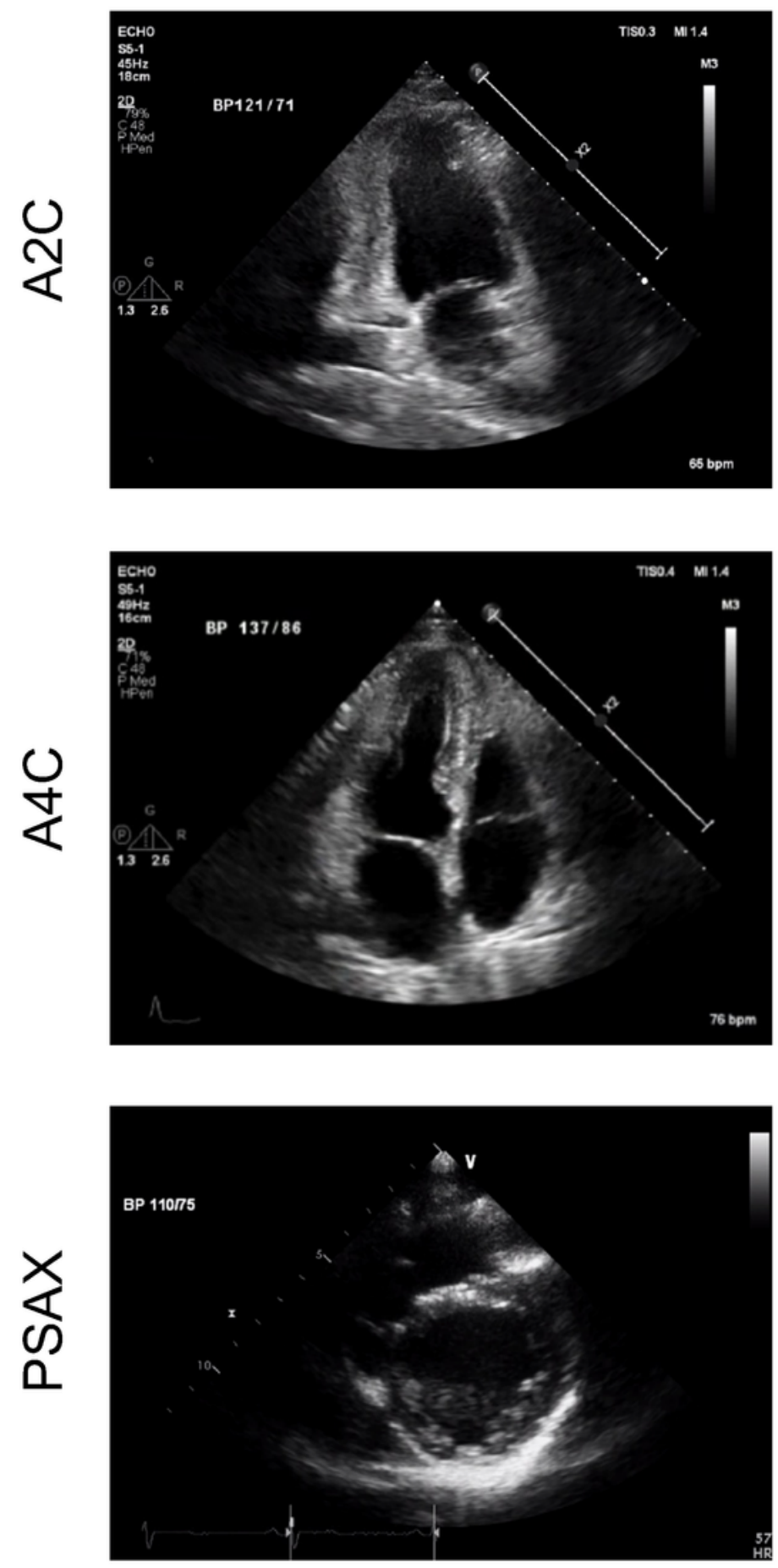

Ground truth
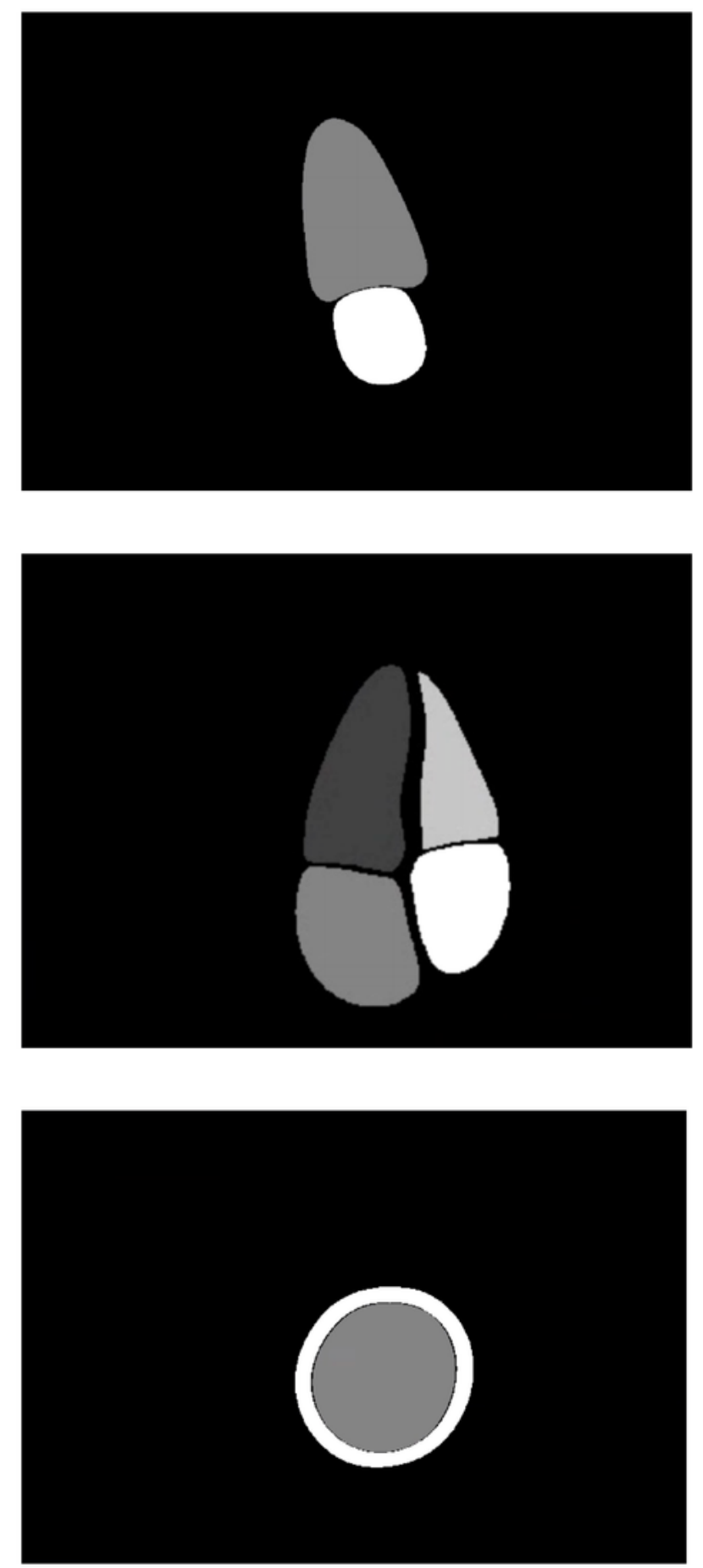

Figure 1

Representative echocardiography images and segmented cardiac chambers by experts. 


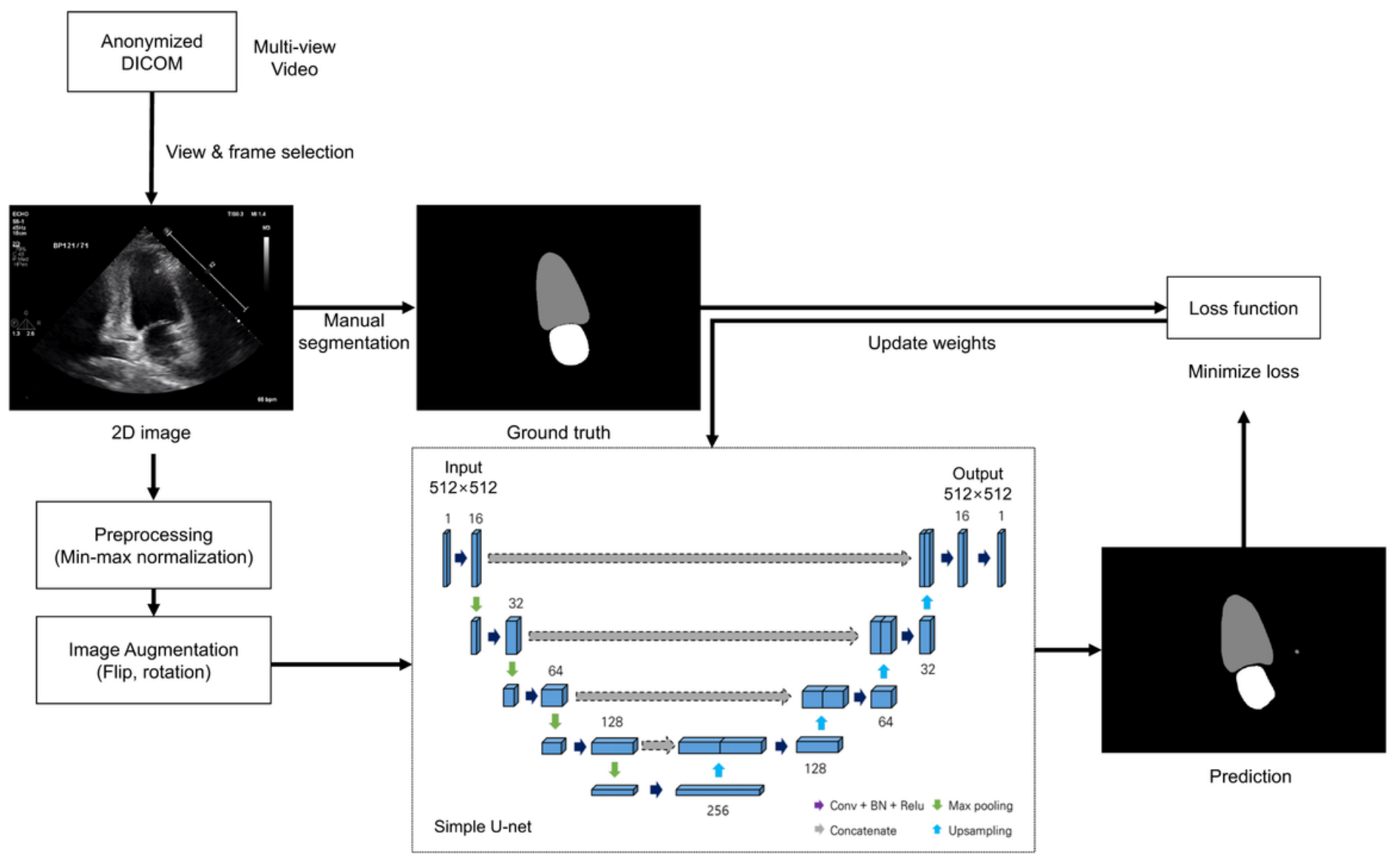

Figure 2

Schematic workflow of the convolutional neural network architecture. 


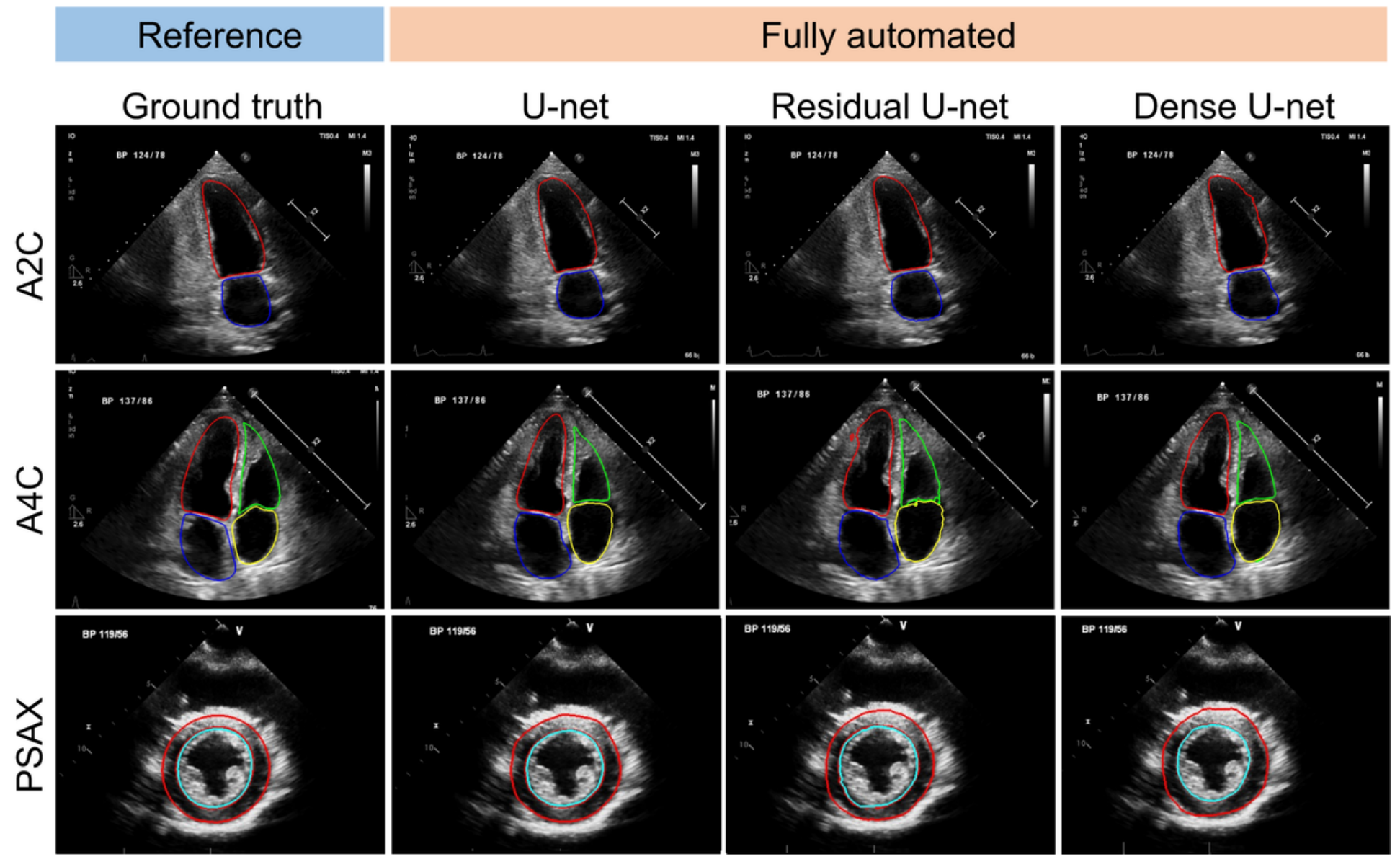

Figure 3

Representative segmentation results of fully automated methods. 

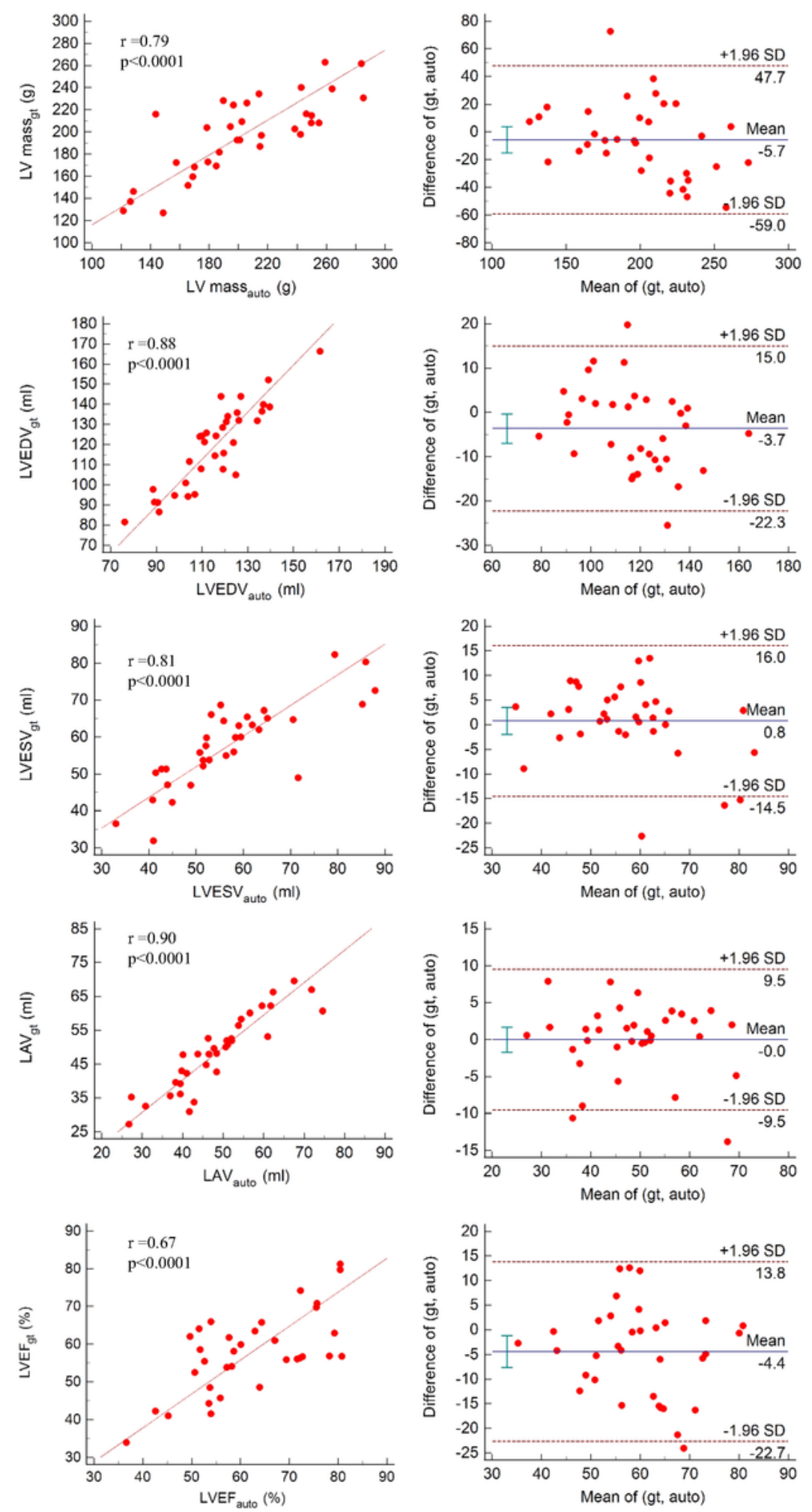

Figure 4

Comparison between automated method and manual measurement of LV volumes, mass, LA volume, and EF: linear regression (left) and Bland-Altman analysis (right) for LV mass, LVEDV, LVESV, LAV, and LVEF from top to bottom. 

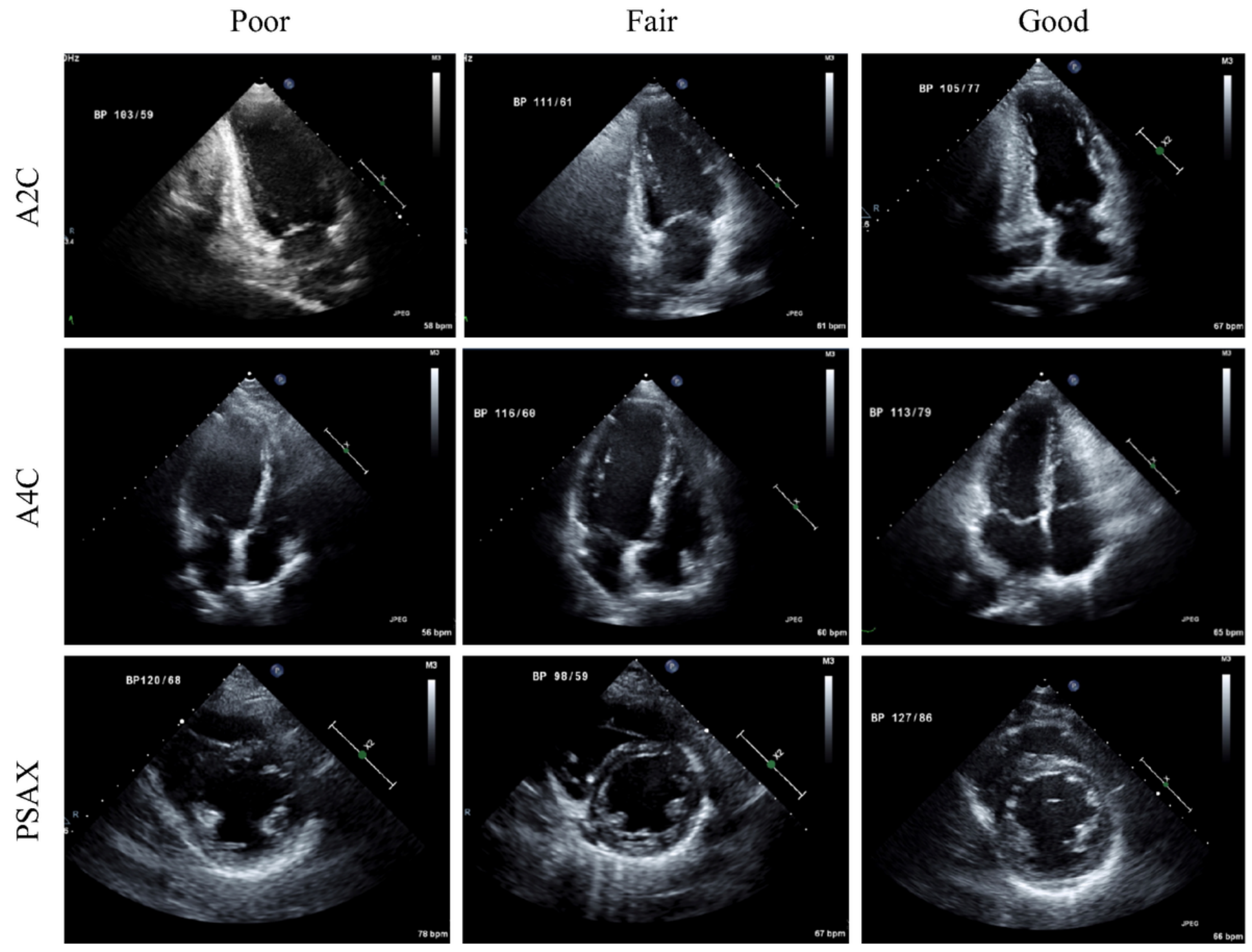

Figure 5

Representative echocardiography image from good to bad image quality. 


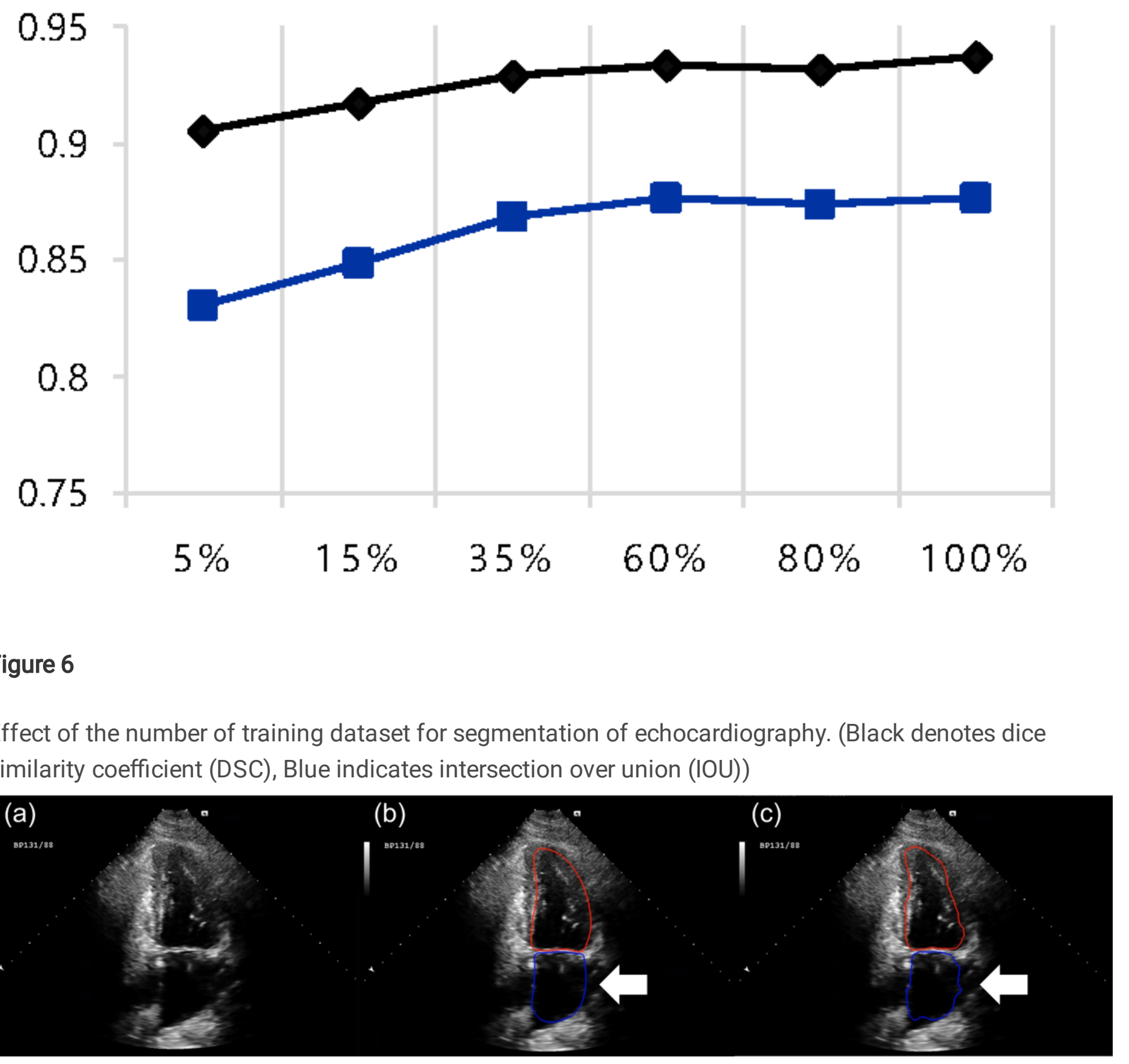

Figure 7

An example of worst prediction result. Left atrium with Incomplete boundary interferes segmentation accuracy. The white arrow indicates incomplete left atrium boundary. (a) the original image (b) ground truth overlaid on image (c) prediction result overlaid on image (red: left ventricle, blue: left atrium) 